\title{
Das Google-Urteil des EuGH und die Entfernungspflicht von Suchmaschinen nach schweizerischem Recht
}

Daniel Hürlimann*

Das Google-Urteil des EuGH verstösst gegen die Informationsfreiheit, die sowohl von der EU-Grundrechtecharta als auch in der EMRK und BV garantiert wird. Mit dem Urteil wird Suchmaschinenbetreibern eine Richterfunktion zugewiesen, die in einem Rechtsstaat nicht an Private delegiert werden darf.

I. Einleitung

II. Das Urteil des EuGH vom 13. Mai 2014

III. Die Beurteilung durch den Generalanwalt

1. Suchmaschinenbetreiber ist kein «für die Verarbeitung Verantwortlicher»

2. Meinungsäusserungs- und Informationsfreiheit geopfert

IV. Offene Fragen

1. Kriterien zur Entfernung

2. Betroffene Dienste

3. Form der Mitteilung

4. Umfang der Entfernung

V. Die Rechtslage in der Schweiz

1. Datenschutzrecht

2. Grundrechte

3. Rechtfertigungsgründe

4. Kartellrecht

VI. Ausblick

1. Weiterzug an den EGMR

2. Rückzug aus EU-Staaten

3. Korrektur durch den Gesetzgeber

4. Alternative Lösungsmöglichkeiten

VII. Fazit

Zitiervorschlag: Daniel Hürlimann, Das Google-Urteil des EuGH und die Entfernungspflicht von Suchmaschinen nach schweizerischem Recht, in: sui-generis 2014, S. 1

URL: $\quad$ sui-generis.ch/1

DOI: $\quad$ https://doi.org/10.21257/sg.1

Dr. iur., Rechtsanwalt, CAS Judikative. Forschungsmitarbeiter an den Universitäten Luzern und Zürich. twitter.com/dhuerlimann Themenbezogene Interessen(-bindung) des Autors: keine

Der Autor dankt Prof. Dr. Astrid Epiney, Prof. Dr. Monika Pfaffinger und MLaw Danielle Schneider für die kritische Durchsicht des Manuskripts. 


\section{Einleitung}

1 Mit einem Recht auf Vergessenwerden «würden entscheidende Rechte wie die Freiheit der Meinungsäusserung und die Informationsfreiheit geopfert» ${ }^{1}$. Gestiitzt auf diese Argumentation hat der Generalanwalt in einem Verfahren gegen Google dem EuGH davor abgeraten, ein solches Recht zu bejahen. Die Warnung wurde nicht erhört: Mit Urteil vom 13. Mai 2014 hat der EuGH festgehalten, dass Google verpflichtet ist, Links mit Informationen zu einer Person zu entfernen, «auch wenn der Name oder die Informationen auf diesen Internetseiten nicht vorher oder gleichzeitig gelöscht werden und gegebenenfalls auch dann, wenn ihre Veröffentlichung auf den Internetseiten als solche rechtmäßig ist.» ${ }^{2}$

2 Konkret ging es in diesem Verfahren um einen Spanier, der sich dagegen wehrte, dass nach einer Google-Suche mit seinem Namen eine bestimmte Seite angezeigt und verlinkt wurde. Diese Seite enthielt die Information, dass sein Grundstück wegen ausstehenden Forderungen der Sozialversicherung versteigert wurde ${ }^{3}$. Die Publikation der Anzeige erfolgte im Jahre 1998 in einer gedruckten Zeitung und wurde später durch den Verleger auch online veröffentlicht ${ }^{4}$.

3 Voraussetzung für die Bejahung einer Verpflichtung zur Entfernung war, dass der Suchmaschinenbetreiber als Verantwortlicher für die Verarbeitung personenbezogener Daten qualifiziert $^{5}$ und dass darüber hinaus der räumliche Anwendungsbereicht der Richtlinie bejaht wurde ${ }^{6}$, obwohl Google nicht offengelegt hat, in welchem Staat sich die fraglichen Server befinden? ${ }^{7}$. Das gestützt auf Datenschutzrecht ergangene Urteil kam unerwartet, zumal die Schlussanträge des Generalanwalts in eine andere Richtung gingen und auch deshalb, weil die Veröffentlichung der fraglichen Information in diesem Fall nicht nur rechtmässig war, sondern sogar auf Anordnung einer staatlichen Behörde erfolgte ${ }^{8}$.

4 Im Folgenden wird zunächst das Urteil des EuGH zusammengefasst (II.), bevor auf die abweichende Beurteilung des Generalanwalts (III.) und auf ein paar offene Fragen (IV.) eingegangen wird. Im Anschluss daran soll die Rechtslage in der Schweiz dargestellt werden (V.), um abschliessend einen Ausblick auf mögliche Entwicklungen (VI.) zu wagen. Bei der vorliegenden Darstellung liegt der Fokus vor dem Hintergrund des EuGH-Urteils auf dem Datenschutzrecht ${ }^{9}$.

\section{Das Urteil des EuGH vom 13. Mai 2014}

5 Ein Spanier erhob 2010 bei der spanischen Datenschutzbehörde gegen die Herausgeberin einer Tagesezeitung sowie gegen Google Spain und Google Inc. eine Beschwerde, mit der er beantragte, die Tageszeitung «anzuweisen, entweder die genannten Seiten zu löschen oder zu ändern, so dass die ihn betreffenden
Schlussanträge des Generalanwalts Niilo Jääskinen vom 25. Juni 2013 in der Rechtssache C-131/12, Rn. 133.

2 Urteil des EuGH C-131/12 vom 13. Mai 2014 (Google Spain SL und Google Inc. gegen Agencia Española de Protección de Datos [AEPD] und Mario Costeja González), Rn. 88.

3 Urteil des EuGH C-131/12 vom 13. Mai 2014, Rn. 14.

4 Schlussanträge des Generalanwalts vom 25. Juni
2013 in der Rechtssache C-131/12, Rn. 18.

Urteil des EuGH vom 13. Mai 2014, Rn. 41.

6 Urteil des EuGH vom 13. Mai 2014, Rn. 60.

7 Urteil des EuGH vom 13. Mai 2014, Rn. 43.

8 Urteil des EuGH vom 13. Mai 2014, Rn. 16.

9 Für die Beurteilung der Haftung von Suchmaschinen aus Urheber-, Marken-, Lauterkeits-, Kartellund Persönlichkeitsrecht siehe Daniel Hürlimann, Suchmaschinenhaftung, Diss. Bern 2012. 
personenbezogen Daten dort nicht mehr angezeigt würden, oder zum Schutz der Daten von bestimmten, von den Suchmaschinen zur Verfügung gestellten technischen Möglichkeiten Gebrauch zu machen. Er beantragte ferner, Google Spain oder Google Inc. anzuweisen, ihn betreffende personenbezogene Daten zu löschen oder zu verbergen, so dass diese weder in den Suchergebnissen noch in Links zu La Vanguardia [eine insbesondere in Katalonien weitverbreitete Tageszeitung ${ }^{10}$ ] erschienen» ${ }^{11}$. Die fraglichen Seiten enthielten eine Anzeige, in der unter Nennung des Namens des Betroffenen auf die Versteigerung eines Grundstücks im Zusammenhang mit einer wegen Forderungen der Sozialversicherung erfolgten Pfändung hingewiesen wurde.

Die spanische Datenschutzbehörde wies die Beschwerde, soweit sie sich gegen die Herausgeberin richtete, mit der Begründung zurück, die Veröffentlichung der betreffenden Informationen durch diese Gesellschaft sei rechtlich gerechtfertigt gewesen, da sie auf Anordnung des Arbeits- und Sozialministeriums und mit dem Ziel einer höchstmöglichen Publizität der Zwangsversteigerung und somit einer höchstmöglichen Zahl an Bietern erfolgt $\operatorname{sei}^{12}$. Soweit sie sich gegen Google Spain und Google Inc. richtete, wurde der Beschwerde hingegen stattgegeben. Die Datenschutzbehörde begründete dies damit, dass Suchmaschinenbetreiber eine Datenverarbeitung vornähmen, für die sie verantwortlich seien, und als Mittler der Informationsgesellschaft fungierten; sie unterlägen deshalb den Datenschutzvorschriften $^{13}$. Gegen diese Entscheidung haben Google Spain und Google Inc. bei der Audiencia Nacional Klage erhoben; die Audiencia Nacional hat das Verfahren ausgesetzt und dem EuGH die Fragen vorgelegt, deren Beantwortung davon abhänge, wie die EU-Datenschutzrichtlinie ${ }^{14}$ im Kontext von Technologien, die nach ihrer Bekanntmachung aufgekommen seien, auszulegen sei.

7 Die insgesamt neun Vorlagefragen der Audiencia Nacional wurdem vom EuGH in vier Abschnitten zu den folgenden Themen beantwortet:

1. sachlicher Anwendungsbereich der Richtlinie,

2. räumlicher Anwendungsbereich der Richtlinie,

3. Umfang der Verantwortlichkeit des Suchmaschinenbetreibers nach der Richtlinie,

4. Umfang der durch die Richtlinie garantierten Rechte der betroffenen Person.

\section{Tätigkeit einer Suchmaschine ist eine Verarbeitung personenbezogener Daten}

8 Die EU-Datenschutzrichtlinie gilt gemäss deren Art. 3 «für die ganz oder teilweise automatisierte Verarbeitung personenbezogener Daten sowie für die nicht automatisierte Verarbeitung personenbezogener Daten, die in einer Datei gespeichert sind oder gespeichert werden sollen.»Bei der Prüfung des sachlichen Anwendungsbereichs hatte der EuGH demzufolge zu untersuchen, ob die Tätigkeit einer Suchmaschine als «Verarbeitung personenbezogener Daten» einzustufen ist ${ }^{15}$. Er bejahte die Frage unter Hinweis darauf, dass mit dem

10 Urteil des EuGH vom 13. Mai 2014, Rn. 14.

11 Urteil des EuGH vom 13. Mai 2014, Rn. 15.

12 Urteil des EuGH vom 13. Mai 2014, Rn. 16.

13 Urteil des EuGH vom 13. Mai 2014, Rn. 17.

14 Richtlinie 95/46/EG des Europäischen Parlaments und des Rates vom 24. Oktober 1995 zum Schutz

natürlicher Personen bei der Verarbeitung personenbezogener Daten und zum freien Datenverkehr (AB1. L 281 S. 31-50).

15 Dieser Begriff ist in Art. 2 lit. b der Richtlinie folgendermassen definiert: «Im Sinne dieser Richtli- 
Betrieb einer Suchmaschine zahlreiche Vorgänge einhergehen, die gemäss Datenschutzrichtlinie explizit als Verarbeitung personenbezogener Daten gelten, namentlich das Erheben, das Auslesen, das Speichern, die Organisation, die Aufbewahrung, die Weitergabe und die Bereitstellung ${ }^{16}$. Daran ändere auch nichts, dass die personenbezogenen Daten bereits im Internet veröffentlicht worden sind und von der Suchmaschine nicht verändert werden ${ }^{17}$.

\section{Auf Werbung ausgerichtete Tochterge- sellschaft genügt für Anwendbarkeit des Rechts des betreffenden Staates}

In einem nächsten Schritt stellte sich die Frage, ob die EU-Datenschutzrichtlinie räumlich anwendbar ist, wenn die eigentliche Verarbeitung (möglicherweise ${ }^{18}$ ) nicht im betreffenden EU-Staat vorgenommen wird, der Suchmaschinenbetreiber dort aber eine für Werbung zuständige Tochtergesellschaft hat. Obwohl diese Frage bei streng grammatikalischer Auslegung auch anders beantwortet werden könnte ${ }^{19}$, wird sie vom EuGH mit der folgenden Begründung bejaht: «die Tätigkeiten des Suchmaschinenbetreibers und die seiner Niederlassung in dem betreffenden Mit- gliedstaat [sind] untrennbar miteinander verbunden, da die die Werbeflächen betreffenden Tätigkeiten das Mittel darstellen, um die in Rede stehende Suchmaschine wirtschaftlich rentabel zu machen, und die Suchmaschine gleichzeitig das Mittel ist, das die Durchführung dieser Tätigkeiten ermöglicht ${ }^{20}$.

\section{Suchmaschinenbetreiber ist auch zur Ent- fernung rechtmässig veröffentlichter In- halte verpflichtet}

10 Nach der Bejahung des sachlichen und räumlichen Anwendungsbereichs der EU-Datenschutzrichtlinie kommt der EuGH zur materiellen Kernfrage, d.h. der Frage nach dem Umfang der Verantwortlichkeit des Suchmaschinenbetreibers. Der Gerichtshof weist zunächst darauf hin, dass die Bestimmungen der Richtlinie, soweit sie Verarbeitungen personenbezogener Daten betreffen, im Licht der Grundrechte auszulegen sind, und hebt insbesondere Art. 7 (Recht auf Achtung des Privatlebens) und 8 (Recht auf Schutz der personenbezogenen Daten) der Charta der Grundrechte der Europäischen Union ${ }^{21}$ hervor.

11 Art. 8 Abs. 2 der Charta hält fest, dass personenbezogene Daten «nur nach Treu und Glau- nie bezeichnet der Ausdruck <Verarbeitung personenbezogener Daten (〈Verarbeitung $\rangle$ ) jeden mit oder ohne Hilfe automatisierter Verfahren ausgeführten Vorgang oder jede Vorgangsreihe im Zusammenhang mit personenbezogenen Daten wie das Erheben, das Speichern, die Organisation, die Aufbewahrung, die Anpassung oder Veränderung, das Auslesen, das Abfragen, die Benutzung, die Weitergabe durch Übermittlung, Verbreitung oder jede andere Form der Bereitstellung, die Kombination oder die Verknüpfung sowie das Sperren, Löschen oder Vernichten;».

16 Urteil des EuGH vom 13. Mai 2014, Rn. 28.

17 Urteil des EuGH vom 13. Mai 2014, Rn. 29 f., mit Verweis auf das Urteil des EuGH C-73/07 vom 16. Dezember 2008 (Tietosuojavaltuutettu gegen Satakunnan Markkinapörssi und Satamedia), Rn. 48 und 49
18 Google Search wird von der Google Inc. mit Sitz in den USA betrieben und gibt nicht bekannt, in welchem Staat sich die Server befinden, «da diese Information aus Wettbewerbsgründen geheimgehalten wird.» Urteil des EuGH vom 13. Mai 2014, Rn. 43.

19 Art. 4 Abs. 1 lit. a der EU-Datenschutzverordnung legt fest, dass jeder Mitgliedstaat die Vorschriften, die er zur Umsetzung dieser Richtlinie erläßt, auf alle Verarbeitungen personenbezogener Daten anwendet, «die im Rahmen der Tätigkeiten einer Niederlassung ausgeführt werden, die der für die Verarbeitung Verantwortliche im Hoheitsgebiet dieses Mitgliedstaats besitzt. [...]» (Hervorhebung hinzugefügt).

20 Urteil des EuGH vom 13. Mai 2014, Rn. 56.

21 Charta der Grundrechte der Europäischen Union (ABl. C 364 vom 18/12/2000, S. 1-22). 
ben für festgelegte Zwecke und mit Einwilligung der betroffenen Person oder auf einer sonstigen gesetzlich geregelten legitimen Grundlage verarbeitet werden» dürfen. Art. 6 Abs. 1 der Richtlinie geht darüber hinaus, indem er verlangt, dass personenbezogene Daten zusätzlich «sachlich richtig und, wenn nötig, auf den neuesten Stand gebracht sind» (lit. d) und «nicht länger, als es für die Realisierung der Zwecke, für die sie erhoben oder weiterverarbeitet werden, erforderlich ist, in einer Form aufbewahrt werden, die die Identifizierung der betroffenen Personen ermöglicht» (lit. e). Sodann regelt Art. 7 der Richtlinie die Zulässigkeit der Verarbeitung von personenbezogenen Daten, wobei für eine von einem Suchmaschinenbetreiber ausgeführte Verarbeitung als Zulässigkeitsgrund Art. 7 lit. f einschlägig ist. Nach dieser Bestimmung darf die Verarbeitung personenbezogener Daten erfolgen, wenn «die Verarbeitung [...] erforderlich [ist] zur Verwirklichung des berechtigten Interesses, das von dem für die Verarbeitung Verantwortlichen oder von dem bzw. den Dritten wahrgenommen wird, denen die Daten übermittelt werden, sofern nicht das Interesse oder die Grundrechte und Grundfreiheiten der betroffenen Person, die gemäß Artikel $1 \mathrm{Ab}$ satz 1 geschützt sind, überwiesen [sic].»

Im Rahmen der Grundrechtsabwägung hält der EuGH zunächst fest, dass die Tätigkeit der Suchmaschinenbetreiber «die Grundrechte auf Achtung des Privatlebens und Schutz personenbezogener Daten erheblich beeinträchtigen [kann], wenn die Suche mit dieser Suchmaschine anhand des Namens einer natürlichen Person durchgeführt wird, da diese Verarbeitung es jedem Internetnutzer ermöglicht, mit der Ergebnisliste einen strukturierten Überblick über die zu der betreffenden Person im Internet zu findenden Informationen zu erhalten, die potenziell zahlreiche Aspekte von deren Privatleben betreffen und ohne die betreffende Suchmaschine nicht oder nur sehr schwer hätten miteinander verknüpft werden können, und somit ein mehr oder weniger detailliertes Profil der Person zu erstellen. Zudem wird die Wirkung des Eingriffs in die genannten Rechte der betroffenen Person noch durch die bedeutende Rolle des Internets und der Suchmaschinen in der modernen Gesellschaft gesteigert, die den in einer Ergebnisliste enthaltenen Informationen Ubiquität verleihen ${ }^{22}$.

13 Leider geht das Urteil auf die gegenüberstehenden Grundrechte nicht in gleicher Weise ein. Die eigentliche Abwägung fällt dann auch erstaunlich kurz aus: Die wirtschaftlichen Interessen des Suchmaschinenbetreibers überwiegen für sich gesehen nie und das Interesse der Allgemeinheit am Zugang zu Informationen überwiegt im Allgemeinen auch nicht - es sei denn, es gehe um Informationen zu einer Person des öffentlichen Lebens. In den Worten des $\mathrm{EuGH}^{23}$ :

«Wegen seiner potenziellen Schwere kann ein solcher Eingriff nicht allein mit dem wirtschaftlichen Interesse des Suchmaschinenbetreibers an der Verarbeitung der Daten gerechtfertigt werden. Da sich die Entfernung von Links aus der Ergebnisliste aber je nach der Information, um die es sich handelt, auf das berechtigte Interesse von potenziell am Zugang zu der Information interessierten Internetnutzern auswirken kann, ist in Situationen wie der des Ausgangsverfahrens ein angemessener Ausgleich u. a. zwischen diesem Interesse und den Grundrechten der 
betroffenen Person aus den Art. 7 und 8 der Charta zu finden. Zwar überwiegen die durch diese Artikel geschützten Rechte der betroffenen Person im Allgemeinen gegenüber dem Interesse der Internetnutzer; der Ausgleich kann in besonders gelagerten Fällen aber von der Art der betreffenden Information, von deren Sensibilität für das Privatleben der betroffenen Person und vom Interesse der Öffentlichkeit am Zugang zu der Information abhängen, das $u$. a. je nach der Rolle, die die Person im öffentlichen Leben spielt, variieren kann.»

Sodann begründet der EuGH überzeugend, warum die Verpflichtung zur Entfernung von Suchtreffern durch den Suchmaschinenbetreiber auch dann möglich sein muss, wenn die betreffenden Webseiten weiterhin online bleiben: Erstens erfolgt durch Suchmaschinen eine zusätzliche Beeinträchtigung der Grundrechte ${ }^{24}$, zweitens unterliegen die Herausgeber dieser Webseiten nicht zwingend dem Unionsrecht ${ }^{25}$, drittens können diese Webseiten unter dem Schutz des Journalistenprivilegs gemäss Art. 9 der Richtlinie stehen ${ }^{26}$ und viertens kann die Interessenabwägung je nachdem, ob es sich um einen Suchmaschinenbetreiber oder den Websitebetreiber handelt, anders ausfallen ${ }^{27}$.
15 Das Zwischenfazit des EuGH lautet demzufolge, dass der Suchmaschinenbetreiber «dazu verpflichtet ist, von der Ergebnisliste, die im Anschluss an eine anhand des Namens einer Person durchgeführte Suche angezeigt wird, Links zu von Dritten veröffentlichten Internetseiten mit Informationen zu dieser Person zu entfernen, auch wenn der Name oder die Informationen auf diesen Internetseiten nicht vorher oder gleichzeitig gelöscht werden und gegebenenfalls auch dann, wenn ihre Veröffentlichung auf den Internetseiten als solche rechtmäßig ist.» ${ }^{28}$

\section{Die Grundrechte der betroffenen Person überwiegen gegenüber dem Interesse der Öffentlichkeit}

16 Schliesslich äussert sich der EuGH zur Frage, ob die betroffene Person nach der Richtlinie verlangen kann, dass Links zu rechtmässig veröffentlichten Webseiten vom Suchmaschinenbetreiber entfernt werden, weil sie wünscht, dass die darin über sie enthaltenen Informationen nach einer gewissen Zeit vergessen werden.

17 Der Gerichtshof hält zunächst unter Verweis auf Art. 6 der EU-Datenschutzrichtlinie fest, «dass auch eine ursprünglich rechtmäßige Verarbeitung sachlich richtiger Daten im
24 Urteil des EuGH vom 13. Mai 2014, Rn. 83.

25 Urteil des EuGH vom 13. Mai 2014, Rn. 84.

26 Urteil des EuGH vom 13. Mai 2014, Rn. 85.

27 Urteil des EuGH vom 13. Mai 2014, Rn. 86; dies deshalb, weil «[d]ie Aufnahme einer Internetseite [...] in die Liste mit den Ergebnissen einer anhand des Namens der betreffenden Person durchgeführten Suche [...] einen stärkeren Eingriff in das Grundrecht auf Achtung des Privatlebens der betroffenen Person darstellen [kann] als die Veröffentlichung durch den Herausgeber der Internetseite.» (Rn. 87).

28 Ungekürzt lautet das Fazit im Urteil des EuGH vom 13. Mai 2014, Rn. 88: «Somit ist auf Frage 2

\begin{abstract}
Buchst. c und d zu antworten, dass Art. 12 Buchst. b und Art. 14 Abs. 1 Buchst. a der Richtlinie 95/46 dahin auszulegen sind, dass der Suchmaschinenbetreiber zur Wahrung der in diesen Bestimmungen vorgesehenen Rechte, sofern deren Voraussetzungen erfüllt sind, dazu verpflichtet ist, von der Ergebnisliste, die im Anschluss an eine anhand des Namens einer Person durchgeführte Suche angezeigt wird, Links zu von Dritten veröffentlichten Internetseiten mit Informationen zu dieser Person zu entfernen, auch wenn der Name oder die Informationen auf diesen Internetseiten nicht vorher oder gleichzeitig gelöscht werden und gegebenenfalls auch dann, wenn ihre Veröffentlichung auf den Internetseiten als solche rechtmäßig ist.»
\end{abstract}


Laufe der Zeit nicht mehr den Bestimmungen der Richtlinie entsprechen kann, wenn die Daten für die Zwecke, für die sie erhoben oder verarbeitet worden sind, nicht mehr erforderlich sind. Das ist insbesondere der Fall, wenn sie diesen Zwecken in Anbetracht der verstrichenen Zeit nicht entsprechen, dafür nicht oder nicht mehr erheblich sind oder darüber hinausgehen ${ }^{29}$. Die Pflicht zur Löschung der betreffenden Informationen und Links der Ergebnisliste auf Antrag der betroffenen Person gelte somit auch für rechtmässig veröffentlichte Internetseiten, die wahrheitsgemässe Informationen enthalten ${ }^{30}$.

Es folgt dann die bereits bekannte Abwägung, die eigentlich keine ist: «Da die betroffene Person in Anbetracht ihrer Grundrechte aus den Art. 7 und 8 der Charta verlangen kann, dass die betreffende Information der breiten Öffentlichkeit nicht mehr durch Einbeziehung in eine derartige Ergebnisliste zur Verfügung gestellt wird, ist [...] davon auszugehen, dass diese Rechte grundsätzlich nicht nur gegenüber dem wirtschaftlichen Interesse des Suchmaschinenbetreibers, sondern auch gegenüber dem Interesse der breiten Öffentlichkeit daran, die Information bei einer anhand des Namens der betroffenen Person durchgeführten Suche zu finden, überwiegen ${ }^{31}$. Auch hier wird als einzige Ausnahme das Interesse an Personen des öffentlichen Lebens genannt. Zudem fällt auf, dass die vom vorlegenden
Gericht aufgeworfene Frage, ob die betroffene Person die Indexierung verhindern $\mathrm{kann}^{32}$, nicht beantwortet wird.

19 Bezogen auf den konkreten Fall hält der Gerichtshof abschliessend fest, dass «die betroffene Person wegen der Sensibilität der in diesen Anzeigen enthaltenen Informationen für ihr Privatleben und weil die ursprüngliche Veröffentlichung der Anzeigen 16 Jahre zurückliegt, ein Recht darauf hat, dass diese Informationen nicht mehr durch eine solche Ergebnisliste mit ihrem Namen verknüpft werden ${ }^{33}$.

\section{Die Beurteilung durch den Generalanwalt}

20 Im Unterschied zum Urteil des EuGH finden sich in den Schlussanträgen des Generalanwalts vom 25. Juni 2013 ausführliche Überlegungen zur grundrechtlichen Dimension dieses Falles ${ }^{34}$.

21 In den Vorbemerkungen weist der Generalanwalt zunächst darauf hin, dass der Gerichtshof gehalten ist, «bei der Auslegung des Anwendungsbereichs der Richtlinie Vernunft walten $\mathrm{zu}$ lassen, mit anderen Worten den Grundsatz der Verhältnismäßigkeit anzuwenden, um unangemessene und übermäßige Rechtsfolgen $\mathrm{zu}$ vermeiden ${ }^{35}$. Sodann verneint er die
29 Urteil des EuGH vom 13. Mai 2014, Rn. 93.

30 Urteil des EuGH vom 13. Mai 2014, Rn. 94.

31 Urteil des EuGH vom 13. Mai 2014, Rn. 97.

32 Die Frage lautet gemäss Urteil des EuGH C131/12 vom 13. Mai 2014, Rn. 20: «Sind das Recht auf Löschung und Sperrung personenbezogener Daten gemäß Art. 12 Buchst. b der Richtlinie 95/46 und das Recht auf Widerspruch gegen eine Verarbeitung gemäß Art. 14 Abs. 1 Buchst. a der Richtlinie 95/46 dahin auszulegen, dass sich die betroffene Person an die Suchmaschinenbetreiber wenden kann, um die Indexierung auf sie bezo- gener Informationen zu verhindern, die auf Websites Dritter veröffentlicht sind, und sie sich hierzu auf ihren Willen berufen kann, dass sie den Internetnutzern nicht bekannt werden, wenn sie der Ansicht ist, dass sie ihr schaden können, oder sie sich wünscht, dass sie vergessen werden, selbst wenn es sich um Informationen handelt, die von Dritten rechtmäßig veröffentlicht wurden?».

33 Urteil des EuGH vom 13. Mai 2014, Rn. 98.

34 Schlussanträge des Generalanwalts vom 25. Juni 2013, Rn. 112-137.

35 Schlussanträge des Generalanwalts vom 25. Juni 2013, Rn. 30. 
Frage, ob die E-Commerce-Richtlinie ${ }^{36}$ auch auf Suchmaschinen anwendbar sei, weist aber gleichzeitig darauf hin, dass ihre Stellung trotzdem «anhand der Rechtsgrundsätze zu prüfen [ist], die für die eingeschränkte Verantwortlichkeit der Internetdiensteanbieter gelten» ${ }^{37}$.

Der Gerichtshof hat in seinem Urteil die Frage der Anwendbarkeit dieser Richtlinie nicht aufgegriffen. Dies erstaunt, zumal die Richtlinie Haftungsfreistellungsregeln für verschiedene «Dienste der Informationsgesellschaft» enthält ${ }^{38}$ und ihre (analoge) Anwendbarkeit auf Suchmaschinen umstritten ist. Verschiedene Mitgliedstaaten der EU haben bei der Umsetzung der Richtlinie in ihr nationales Recht explizit Haftungsfreistellungen auch für Suchmaschinen erlassen ${ }^{39}$.

\section{Suchmaschinenbetreiber ist kein «für die Verarbeitung Verantwortlicher»}

Im Weiteren hat sich der Generalanwalt ausführlich zum räumlichen und sachlichen An- wendungsbereich der EU-Datenschutzrichtlinie und schliesslich zur Frage bezüglich eines der betroffenen Person zustehenden «Rechts auf Vergessenwerden» geäussert. Während das Gericht seiner Einschätzung zum räumlichen Anwendungsbereich folgte ${ }^{40}$, wich es bereits beim sachlichen Anwendungsbereich von den Anträgen des Generalanwalts, der diesen verneint, ab. Da es sich um ein Vorabentscheidungsverfahren zur Auslegung der Datenschutzrichtlinie handelt, mussten die Fragen auch hier an einzelnen Begriffen der entsprechenden Bestimmungen aufgehängt werden. Zunächst wird in den Schlussanträgen die Anwendbarkeit des Begriffs «personenbezogene Daten» auf einen Suchmaschinenbetreiber untersucht und ohne weiteres bejaht $^{41}$.

24 Eine erste zentrale Abweichung zum Urteil des EuGH findet sich dann aber in der Auffassung des Generalanwalts, wonach ein Suchmaschinenbetreiber hinsichtlich personenbezogener Daten auf Quellenwebseiten Dritter kein «für die Verarbeitung Verantwortlicher»
36 Richtlinie 2000/31/EG des Europäischen Parlaments und des Rates vom 8. Juni 2000 über bestimmte rechtliche Aspekte der Dienste der Informationsgesellschaft, insbesondere des elektronischen Geschäftsverkehrs, im Binnenmarkt («Richtlinie über den elektronischen Geschäftsverkehr») (AB1. L 178, S. 1-16).

37 Schlussanträge des Generalanwalts vom 25. Juni 2013, Rn. 38.

38 Art. 12 («Reine Durchleitung», u.a. Access Provider), Art. 13 («Caching») und Art. 14 («Hosting») E-Commerce-Richtlinie.

39 Erster Bericht über die Anwendung der Richtlinie 2000/31/EG des Europäischen Parlaments und des Rates vom 8. Juni 2000 über bestimmte rechtliche Aspekte der Dienste der Informationsgesellschaft, insbesondere des elektronischen Geschäftsverkehrs, im Binnenmarkt, Fn. 69: «Spanien und Portugal haben sich hinsichtlich Suchmaschinen und Hyperlinks für das Vorbild des Artikels 14 entschieden, während Österreich und Liechtenstein Artikel 12 als Vorbild für Suchmaschinen und Artikel 14 für Hyperlinks gewählt haben.» Hinzu kommt Ungarn, das die E-Commerce-Richtlinie mit dem «2001. évi CVIII. törvény az elektronikus kereskedelmi szolgáltatások, valamint az információs társadalommal összefüggõ szolgáltatások egyes kérdéseirõl» (Act CVIII of 2001 on certain issues of electronic commerce services and information society services) umgesetzt und dabei auch die Haftung von Suchmaschinen analog den Bestimmungen für Hosting-Provider geregelt hat.

40 Schlussanträge des Generalanwalts vom 25. Juni 2013, Rn. 68; Urteil des EuGH vom 13. Mai 2014, Rn. 60.

41 Schlussanträge des Generalanwalts vom 25. Juni 2013, Rn. 75: «Es versteht sich von selbst, dass die in den vorstehenden Nummern dargestellten Vorgänge als Verarbeitungen der personenbezogenen Daten gelten, die sich auf den von der Suchmaschine kopierten, indexierten, gespeicherten und angezeigten Quellenwebseiten befinden. Insbesondere umfassen sie das Erheben, das Speichern, die Organisation und die Aufbewahrung solcher personenbezogenen Daten [...]». 
ist $^{42}$. Der Generalanwalt verweist in seiner Begründung zur Verneinung dieser Frage auf ein anschauliches Beispiel:

«Zu denken ist etwa an einen Professor für Europarecht, der von der Website des Gerichtshofs die wesentliche Rechtsprechung des Gerichtshofs auf seinen Laptop herunterlädt. Nach der Richtlinie lässt sich dieser Professor als ein «für die Verarbeitung Verantwortlicher» im Hinblick auf personenbezogene Daten bezeichnen, die von einem Dritten stammen. Der Professor besitzt Dateien mit personenbezogenen Daten, die bei der Suche und Abfrage im Rahmen von nicht ausschließlich persönlichen oder familiären Tätigkeiten automatisiert verarbeitet werden. Tatsächlich dürfte heutzutage wohl jeder, der eine Zeitung auf einem Tablet liest oder soziale Medien auf einem Smartphone verfolgt, eine Verarbeitung personenbezogener Daten mit Hilfe automatisierter Verfahren vornehmen und könnte in den Anwendungsbereich der Richtlinie fallen, soweit dieser Vorgang in nicht ausschließlich privater Eigenschaft ausgeführt wird» ${ }^{43}$.

«Entscheidet der in meinem obigen Beispiel genannte Professor für Europarecht über die Zwecke und Mittel der Verarbeitung von personenbezogenen Daten, die in den auf seinen Laptop heruntergeladenen Urteilen des Gerichtshofs enthalten sind? Die Feststellung der Artikel-29-Datenschutzgruppe $\left[{ }^{44}\right]$, der zufolge $\ll[\mathrm{s}]$ treng genommen ... die Benutzer des Suchmaschinendienstes ebenfalls als für die Verarbeitung Verantwortliche angesehen werden [könnten]), zeigt, zu welch unsinnigen Ergebnissen eine nicht hinterfragte wortwörtliche Auslegung der Richtlinie im Kontext des Internets führen kann. Der Gerichtshof darf keiner Auslegung folgen, die praktisch jede Person, die ein Smartphone, ein Tablet oder einen Laptop besitzt, zu einem für die Verarbeitung von im Internet veröffentlichten personenbezogenen Daten Verantwortlichen $\operatorname{macht»}{ }^{45}$.

25 Der Generalanwalt schlägt zur Lösung des Problems folgendes Verständnis des Begriffs «für die Verarbeitung Verantwortlicher» vor: Der für die Verarbeitung Verantwortliche muss die Verantwortung für die personenbezogenen Daten tragen, d.h. ihm muss «die Existenz einer bestimmten definierten Kategorie von Informationen, die personenbezogene Daten darstellen», nicht nur bekannt sein, er muss diese Daten darüber hinaus in ihrer Eigenschaft als personenbezogene Daten verarbeiten wollen ${ }^{46}$. Für diese Auffassung sprächen sowohl die Systematik der Richtlinie, als auch die meisten Sprachfassungen und schliesslich die Ausgestaltung der einzelnen Pflichten, die die Richtlinie dem für die Verarbeitung Verantwortlichen auferlegt ${ }^{47}$. Gestützt auf diese Auslegung kommt der Generalanwalt zum Ergebnis, dass ein Suchmaschinenbetreiber hinsichtlich personenbezogener Daten auf Quellenwebseiten Dritter kein für die Verarbeitung Verantwortlicher ist, weil
42 Schlussanträge des Generalanwalts vom 25. Juni 2013, Rn. 83 ff.

43 Schlussanträge des Generalanwalts vom 25. Juni 2013, Rn. 29.

44 Die Artikel-29-Datenschutzgruppe wurde im Rahmen der EU-Datenschutzrichtlinie eingerichtet. Sie ist eine beratende und unabhängige Instanz.
45 Schlussanträge des Generalanwalts vom 25. Juni 2013, Rn. 81.

46 Schlussanträge des Generalanwalts vom 25. Juni 2013, Rn. 82.

47 Schlussanträge des Generalanwalts vom 25. Juni 2013, Rn. 82. 
bei der Verarbeitung von dieser Seiten zum Zwecke des Durchsuchens, Analysierens und Indexierens personenbezogene Daten nicht in besonderer Weise hervorstechen ${ }^{48}$.

Nach Ansicht des Generalanwalts wäre vom Grundsatz der Qualifikation des Suchmaschinenbetreibers als nicht für die Verarbeitung Verantwortlicher eine Ausnahme zu machen, wenn dieser die exclusion codes in den Quellenwebseiten nicht beachtet ${ }^{49}$. Es handelt sich dabei um eine technische Möglichkeit zur Nichterfassung von Websites. Dazu wird im Stammverzeichnis der Domain eine Textdatei mit Namen robots.txt angelegt, welche nach einem vorgegebenen Schema abgefasste Anweisungen an Suchmaschinen enthält. Auf diesem Weg können einzelne Verzeichnisse und Verzeichnisbäume oder auch der gesamte Inhalt einer Domain der Erfassung durch alle oder einzeln definierte Suchmaschinen entzogen werden ${ }^{50}$.

\section{Meinungsäusserungs- und Informations- freiheit geopfert}

27 Anders als das Gericht beurteilt der Generalanwalt auch die letzte und zentrale Frage, d.h. die Verantwortlichkeit des Suchmaschinenbetreibers. Er verzichtet dabei auch auf die schwer verständliche Unterscheidung zwischen dem Umfang der Verantwortlichkeit des Suchmaschinenbetreibers nach der EUDatenschutzrichtlinie ${ }^{51}$ und dem Umfang der durch die Richtlinie garantierten Rechte der

betroffenen Person ${ }^{52}$.

28 dass der Suchmaschinenbetreiber, sofern er als für die Verarbeitung Verantwortlicher qualifiziert würde, «seine Funktion als Vermittler zwischen den Nutzern und dem Urheber aufgeben und die Verantwortung für den Inhalt der Quellenwebseite übernehmen und erforderlichenfalls diesen Inhalt zensieren [müsste], indem er den Zugriff darauf verhindert oder beschränkt» ${ }^{53}$.

29 Sodann verneint der Generalanwalt die Existenz eines Rechts auf Vergessenwerden mit der Begründung, dass ein solches als wichtige rechtliche Neuerung im Vorschlag der EUKommission für eine Datenschutz-Grundverordnung $^{54}$ vorgesehen ist und dementsprechend im Urteilszeitpunkt noch nicht existieren $\mathrm{kann}^{55}$. Im Anschluss daran prüft er, ob diese Auslegung der Bestimmungen mit der Charta der Grundrechte der Europäischen Union vereinbar ist. Nach einer kurzen Darstellung des Rechts auf Schutz personenbezogener Daten (Art. 8 der Charta) und auf Achtung des Privat- und Familienlebens (Art. 7 der Charta) geht er auf die Meinungsäusserungs- und Informationsfreiheit (Art. 11 der Charta) ein, die im Urteil des EuGH nicht erwähnt werden. Art. 11 der Charta schützt nicht nur das Recht der Internetnutzer, im Internet verfügbare Informationen zu suchen und $\mathrm{zu}$
48 Schlussanträge des Generalanwalts vom 25. Juni 2013, Rn. 84.

49 Schlussanträge des Generalanwalts vom 25. Juni 2013, Rn. 91 ff.

50 Weiterführend: Suchmaschinenhaftung, Diss. Bern 2012, § 5, I, 4. Zur Begründung einer entsprechenden Sorgfaltspflicht der Suchmaschinenbetreiber hinsichtlich der Beachtung von exclusion codes: ebenda, $\S 11$, I, 2.

51 Urteil des EuGH vom 13. Mai 2014, Rn. 62-88.
52 Urteil des EuGH vom 13. Mai 2014, Rn. 89-99.

53 Schlussanträge des Generalanwalts vom 25. Juni 2013, Rn. 109.

54 Vorschlag für eine Verordnung des Europäischen Parlaments und des Rates zum Schutz natürlicher Personen bei der Verarbeitung personenbezogener Daten und zum freien Datenverkehr (DatenschutzGrundverordnung) (KOM[2012] 11 endgültig).

55 Schlussanträge des Generalanwalts vom 25. Juni 2013, Rn. 110. 
empfangen, sondern auch die Meinungsäusserungsfreiheit der Webseitenurheber ${ }^{56}$. Die Suchmaschinenbetreiber ihrerseits beabsichtigen, Werbeeinnahmen zu erzielen und machen daher von der unternehmerischen Freiheit (Art. 16 der Charta) Gebrauch ${ }^{57}$.

Im Unterschied zum EuGH nimmt der Generalanwalt in seinen Schlussanträgen eine Abwägung der einenader gegenüberstehenden Grundrechte vor. Dabei verweist er zunächst auf ein Urteil des EGMR, gemäss dem auch eine Beschränkung der Wiedergabe von Informationen, die bereits in die öffentliche Sphäre gelangt sind, unter bestimmten Umständen gerechtfertigt sein $\mathrm{kann}^{58}$. Demgegenüber hebt er hervor, dass die mittels einer Suchmaschine betriebene Suche zu den wichtigsten Formen der Ausübung des Grundrechts auf Informationsfreiheit gehört ${ }^{59}$. Dann folgen die zentralen Erwägungen:

«Angesichts der besonders komplexen und schwierigen Grundrechtskonstellation im vorliegenden Fall lässt es sich nicht rechtfertigen, die nach Maßgabe der Richtlinie bestehende Rechtsstellung der betroffenen Personen zu verstärken und um ein Recht auf Vergessenwerden zu ergänzen. Andernfalls würden entscheidende Rechte wie die Freiheit der Meinungsäußerung und die Informationsfreiheit geopfert. Ich möchte dem Gerichtshof auch abraten, in seinem Urteil zu dem Ergebnis zu gelangen, dass diese einander widerstreitenden Interessen im jeweiligen Einzelfall auf zufriedenstellende Weise in ein Gleichgewicht gebracht werden können und dass die Entscheidung dem Internetsuchmaschinen-Diensteanbieter überlassen bleibt. Derartige Verfahren zur Meldung und Entfernung, sollte der Gerichtshof sie vorschreiben, werden wahrscheinlich entweder zu einer automatischen Löschung von Links zu beanstandeten Inhalten oder zu einer von den beliebtesten und wichtigsten Internetsuchmaschinen-Diensteanbietern nicht zu bewältigenden Anzahl von entsprechenden Anträgen führen. [...]

Vor allem sollten die Internetsuchmaschinen-Diensteanbieter nicht mit einer solchen Pflicht belastet werden. Es käme zu einem Eingriff in die Freiheit der Meinungsäußerung des Webseitenurhebers, der in einem solchen Fall ohne angemessenen Rechtsschutz bliebe, da ein ungeregeltes Verfahren zur Meldung und Entfernung eine privatrechtliche Angelegenheit zwischen der betroffenen Person und dem Suchmaschinen-Diensteanbieter wäre. Dies liefe auf eine Zensur der vom Urheber veröffentlichten Inhalte durch einen Privaten hinaus. Auf einem ganz anderen Blatt steht hingegen, dass den Staaten die Handlungspflicht obliegt, gegen einen das Recht auf Privatleben verletzenden Verleger einen wirksamen Rechtsbehelf vorzusehen, der im Kontext des Internets gegen den Webseitenurheber gerichtet wäre.» ${ }^{60}$

31 Diese Überlegungen werden vom EuGH in seinem Urteil mit keinem Wort erwähnt.
56 Schlussanträge des Generalanwalts vom 25. Juni 2013, Rn. 121 f.

57 Schlussanträge des Generalanwalts vom 25. Juni 2013, Rn. 124.

58 Schlussanträge des Generalanwalts vom 25. Juni 2013, Rn. 127, mit Verweis auf das Urteil des
EGMR 24061/04 vom 16. Dezember 2010 (Aleksey Ovchinnikov gegen Russland), § 50 .

59

Schlussanträge des Generalanwalts vom 25. Juni 2013, Rn. 131.

60 Schlussanträge des Generalanwalts vom 25. Juni 2013, Rn. 133 f. (Hervorhebung hinzugefügt). 


\section{Offene Fragen}

32 Nach dem Urteil des EuGH stellt sich eine ganze Reihe von Fragen, von denen im Folgenden einige herausgegriffen werden.

\section{Kriterien zur Entfernung}

33 Der EuGH argumentiert bei der Bejahung des Rechts auf Löschung des beanstandeten Treffers durch den Suchmaschinenbetreiber nebst der Sensibilität der Information damit, dass die ursprüngliche Veröffentlichung der Anzeigen 16 Jahre zurückliegt ${ }^{61}$. Damit stellt sich die Frage, ob der Zugang zu Informationen ab einem gewissen Alter generell weniger stark von der Informationsfreiheit geschützt wird. Sodann bleibt auch offen, ob nur eindeutig negative Informationen auf Verlangen zu entfernen sind, oder ob z.B. auch die Verlinkung einer Seite, die Informationen über das hohe Einkommen einer Person enthält, unterbunden werden kann.

Ein weiteres Problem ist darin zu erblicken, dass eine Seite gleichzeitig unerwünschte und erwünschte Informationen enthalten kann. Vor diesem Hintergrund ist auch darauf hinzuweisen, dass ein kartellrechtlicher Anspruch auf Aufnahme in den Index marktbeherrschender Suchmaschinen besteht ${ }^{62}$. Schliesslich enthält das Urteil auch kaum Leitlinien für die durch den Suchmaschinenbetreiber vorzunehmende Güterabwägung. Das Gericht nennt die Rolle der betreffenden Person im öffentlichen Leben als einen möglichen besonderen Grund, der das Interesse der breiten Öffentlichkeit am Zugang zu der Information als gewichtiger erscheinen lässt.
Wie verhält es sich nun aber z.B. beim Zugang zu einer Information über ein Strafverfahren? Nach Angaben von Google stehen 12 Prozent der Löschanfragen im Zusammenhang mit Festnahmen wegen Kinderpornografie ${ }^{63}$.

\section{Betroffene Dienste}

35 Im Nachgang zum Urteil wurde darüber diskutiert, welche Auswirkungen dieses auf Dienste wie Twitter, Facebook oder auch Wikipedia haben wird. Hierzu ist zunächst festzuhalten, dass im Urteil jeweils von der Ergebnisliste, die im Anschluss an eine anhand des Namens durchgeführte Suche angezeigt wird, die Rede ist. Suchfunktionen und Ergebnislisten werden aber von zahlreichen Diensten der Informationsgesellschaft angeboten, sodass eine Geltung des Urteils z.B. für Twitter und Facebook prima vista naheliegend erscheint.

36 Schwieriger zu beantworten ist dagegen die Frage, welche Auswirkungen das Urteil auf Wikipedia haben wird, zumal auch hier im Anschluss an eine Namenssuche Ergebnislisten angezeigt werden ${ }^{64}$, sofern der Name innerhalb von Wikipedia nicht einmalig ist. Im letzteren Fall wird man dagegen direkt zum entsprechenden Artikel weitergeleitet ${ }^{65}$. Die Anwendbarkeit der Entscheidung auf Wikipedia kann aber kaum davon abhängig gemacht werden, ob der Weg zu einer sensiblen Information über eine Ergebnisliste führt oder nicht. Insofern sind die Sorgen des WikipediaGründers ${ }^{66}$ nicht unberechtigt.
61 Urteil des EuGH vom 13. Mai 2014, Rn. 98.

62 Suchmaschinenhaftung, Diss. Bern 2012, § 11, III.

63 Siehe dazu den Beitrag vom 10. Juni 2014 auf golem.de: Google will auf gelöschte Links hinweisen.

64 Beispiel: http://de.wikipedia.org/wiki/Hans_Meier.
65 Beispiel: http://de.wikipedia.org/wiki/Till_Lindemann.

66 Siehe dazu den Tweet von Jimmy Wales vom 13 Mai 2014: «When will a European Court demand that Wikipedia censor an article with truthful information because an individual doesn't like it?». 
Während es sich bei Twitter, Facebook und Wikipedia um Dienste mit einer grossen Reichweite handelt, ist fraglich, welche Auswirkungen das Urteil auf Suchmaschinen oder Dienste mit Suchfunktionen mit kleinerer Reichweise zeitigen wird. Der EuGH argumetiert in nachvollziehbarer Weise damit, dass die Wirkung des Grundrechtseingriffs durch die bedeutende Rolle des Internets und der Suchmaschinen in der modernen Gesellschaft gesteigert wird ${ }^{67}$. Dies gilt aber nur für Suchmaschinen mit einer gewissen Reichweite, d.h. mit einem grossen Marktanteil; andernfalls würde auch die Suchfunktion der im Verfahren betroffenen Tagesezeitung darunter fallen. Betrachtet man nun die Marktanteile von Suchmaschinen in Europa, ist die Vormachtstellung von Google $\left(92 \%{ }^{68}\right)$ noch viel deutlicher als im weltweiten Vergleich (ca. $69 \%{ }^{69}$ ). Offen ist, inwiefern das Urteil auch für die nächstgrösseren Suchmaschinen wie z.B. Bing oder Yahoo gilt, deren Marktanteil in Europa im tiefen einstelligen Prozentbereich liegt.

Gänzlich infrage gestellt ist schliesslich das Geschäftsmodell von Personensuchmaschinen wie z.B. yasni.ch oder vebidoo.de (123people hat seine Tätigkeit im April 2014 eingestellt ${ }^{70}$ ).

\section{Form der Mitteilung}

39

In seinem Urteil hat sich der EuGH nicht dazu geäussert, auf welchem Weg Löschanträge an die Suchmaschinenbetreiber zu übermitteln sind. Naheliegend ist der Versand einer entsprechenden Anfrage per E-Mail. Für den
Suchmaschinenbetreiber dürfte es aber von Interesse sein, solche Anträge zu bündeln, indem er ein entsprechendes Online-Formular bereitstellt. Sofern er dies tut, stellt sich in einem weiteren Schritt die Frage, ob auf anderem Weg eingereichte Anträge ebenfalls bearbeitet werden müssen oder nicht. M.E. kann der Suchmaschinenbetreiber löschwillige Nutzer auf sein Online-Formular verweisen, sofern dieses einfach auszufüllen ist und nicht standardmässig unnötige Angaben wie z.B. ein Geburtsdatum verlangt werden ${ }^{71}$.

40 Vor diesem Hintergrund ist fraglich, ob Google für den Identitätsnachweis ein identifizierendes Dokument für obligatorisch erklären $\operatorname{darf}^{72}$ oder ob nicht z.B. der Nachweis, dass man über eine entsprechend E-Mail-Adresse verfügt, genügen müsste.

41 Nachdem der EuGH eine Entfernungspflicht bejaht hat und Google ein entsprechendes Formular anbietet, wird es voraussichtlich zahlreiche Entfernungsanträge geben, deren eigentliches Ziel die vollständige Entfernung aus dem Internet sein müsste. Wenn z.B. jemand im Rahmen einer beruflichen Neuorientierung ein Profil auf der Webseite einer Modelagentur eingerichtet, sich dann aber später doch gegen diese Richtung entschieden hat, wird er den Auftritt auf dieser Seite womöglich bereuen. Der naheliegendste Weg wäre eine entsprechende Aufforderung an die Betreiberin der Modelagentur. Bequemer ist jedoch das Ausfüllen des Entfernungsformulars von Google und nach dem Urteil des EuGH wird dieser Entfernungsantrag unabhängig

71 Suchmaschinenhaftung, Diss. Bern 2012, $\S 12$, II, 1.

72 Vgl. dazu das Online-Formular «Antrag auf Entfernung aus den Suchergebnissen gemäß Europäischem Datenschutzrecht». 
von einer (in diesem Beispiel klar bestehenden und grundsätzlich durchsetzbaren ${ }^{73}$ ) Entfernungspflicht des Webseitenbetreibers bearbeitet werden müssen. Damit wird der Bote für den Inhalt seiner Information haftbar gemacht und dies hat zwangsläufig unerwünschte Auswirkungen auf die Neutralität des Boten. Diese Bedenken können nicht mit dem Hinweis auf die ohnehin fragwürdige Neutralität von Google in den Wind geschlagen werden. Denn es ist etwas gänzlich anderes, ob man seine eigenen Leistungen in ein gutes Licht rückt $^{74}$ oder ob man über die Auffindbarkeit sämtlicher Webseiten zu urteilen hat.

\section{Umfang der Entfernung}

42 Suchmaschinen funktionieren (vereinfacht dargestellt ${ }^{75}$ ) nach dem Prinzip, dass sie von allen Seiten eine Kopie anfertigen (Crawling), dann eine Tabelle mit allen Begriffen erstellen und in dieser vermerken, welcher Begriff auf welchen Seiten vorkommt (Indexing). Gibt man einen Suchbegriff ein, erhält der Nutzer eine Trefferliste von Links auf Seiten, die den gesuchten Begriff enthalten (bzw. im Moment der Crawlings enthalten haben). Diese Trefferliste wird mithilfe ausgeklügelter Algorithmen in eine bestimmte Reihenfolge gebracht, mit dem Ziel, die relevantesten Treffer zuoberst anzugeigen (Ranking).
43 Wird ein Suchmaschinenbetreiber nun verpflichtet, bestimmte Links nicht mehr anzuzeigen, ist es technisch gesehen am naheliegendsten, die entsprechende Seite aus dem Index zu entfernen und den Crawler so zu konfigurieren, dass er sie in Zukunft nicht mehr berücksichtigt. Damit wird die entsprechende Seite in keiner Trefferliste mehr erscheinen. Der EuGH hat die Verpflichtung des Suchmaschinenbetreibers jedoch enger gefasst: Das Recht der betroffenen Person überwiegt «grundsätzlich [...] gegenüber dem Interesse der breiten Öffentlichkeit am Zugang zu der Information bei einer anhand des Namens der betroffenen Person durchgeführten Suche» ${ }^{76}$. Die Löschpflicht bezieht sich also nur auf die im Anschluss an eine Namenssuche angezeigte Trefferliste. Programmiertechnisch ist dies mit mehr Aufwand verbunden als eine Entfernung aus dem Index, wird aber von Google ${ }^{77}$ und voraussichtlich auch Microsoft Bing $^{78}$ so umgesetzt. Eine andere Frage ist, ob auch kleinere Suchmaschinenbetreiber die Kapazität haben und dazu gewillt sind, nicht generell die beanstandeten Seiten aus dem Index zu nehmen.

44 Schliesslich stellt sich auch die Frage, ob die beanstandeten Suchtreffer nur auf bestimmten
73 Im genannten Beispiel hat der Betreiber der Modelagentur seinen Sitz in der Schweiz und die Entfernungspflicht besteht unabhängig von allfälligen vertraglichen Verpflichtungen, zumal alles andere gegen das Verbot übermässiger Bindung nach Art. 27 Abs. 2 ZGB verstossen würde.

74 Siehe dazu heise.de vom 17. Juni 2014 (Weitere EU-Kartellbeschwerde gegen Google) sowie kress.de vom 24. Juni 2014 (Presseverlage und VG Media machen Druck: Beschwerde beim Bundeskartellamt gegen Google).

75 Für eine ausführlichere Beschreibung der Funktionsweise von Suchmaschinen: Suchmaschinenhaftung, Diss. Bern 2012, § 5.

76 Urteil des EuGH vom 13. Mai 2014, Rn. 99.

77 Beispiel: Google-Suche nach «daniel huerlimann»; am Ende der Seite steht: «Einige Ergebnisse wurden möglicherweise aufgrund der Bestimmungen des europäischen Datenschutzgesetzes entfernt.» Dieser Hinweis wird gemäss den häufig gestellten Fragen «in Europa bei der Suche nach den meisten Namen an[gezeigt] und nicht nur bei Seiten, die von einer Entfernung betroffen sind.»

78 onlinehelp.microsoft.com zum Thema Einreichung von Anträgen auf Löschung von personenbezogenen Daten in der EU: «Wir arbeiten derzeit an einem speziellen Verfahren für die Bewohner der Europäischen Union, womit sie die Löschung bestimmter personenbezogener Suchergebnisse auf Bing, die bei einer Suche nach ihren Namen erscheinen, beantragen können.» 
Länderseiten oder im gesamten EU-Raum oder gar weltweit zu entfernen sind. Auch wenn ein «Recht auf Vergessenwerden» diesen $\mathrm{Na}$ men - wenn überhaupt - nur dann verdienen würde, wenn die Treffer auf allen Länderseiten einer Suchmaschine entfernt würden, ist unklar, ob eine entsprechende Verpflichtung aus dem Urteil abgeleitet werden kann. Medienberichten zufolge plant Google die Löschung von Links in den EU- und EWR-Staaten und in der Schweiz, nicht aber etwa in der Domain google.com ${ }^{79}$. Nach der Argumentation von Google würde die Entfernung auf allen Länderseiten dazu führen, dass sich die Löschpflichten aus allen Jurisdiktionen summieren würden. Damit wären letztlich nur noch diejenigen Seiten über Suchmaschinen auffindbar, die von sämtlichen Regimes weltweit toleriert werden.

\section{Die Rechtslage in der Schweiz}

\section{Datenschutzrecht}

45 Das schweizerische Datenschutzgesetz ${ }^{80}$ enthält mit der EU-Datenschutzverordnung vergleichbare Bestimmungen. Der EuGH stützt sich in seinem Urteil in erster Linie auf Art. 12 lit. $\mathrm{b}$ und Art. 14 Abs. 1 lit. a der EU-Datenschutzrichtlinie. Art. 12 lit. b der Richtlinie hält fest, dass jede betroffene Person das Recht hat, vom für die Verarbeitung Verantwortlichen die Berichtigung, Löschung oder Sperrung von Daten zu erhalten, wenn deren Verarbeitung nicht den Bestimmungen der Richtlinie entspricht. Die Berichtigung, Löschung oder Sperrung von Daten ist in der Schweiz in Art. 15 Abs. 1 DSG verankert, wobei via Art. 12 DSG auf die Bearbeitungsgrundsätze in Art. 4, 5 Abs. 1 und 7 Abs. 1
DSG verwiesen wird. Gemäss Art. 15 DSG richten sich die Klagen nach Art. 28, 28a sowie $281 \mathrm{ZGB}$, womit sie «gegen jeden, der an der Verletzung mitwirkt» (Art. 28 ZGB) und damit auch gegen Suchmaschinenbetreiber gerichtet werden können.

46 In Art. 14 Abs. 1 lit. a der Richtlinie ist das Widerspruchsrecht der betroffenen Person verankert. Diese kann «jederzeit aus überwiegenden, schutzwürdigen, sich aus ihrer besonderen Situation ergebenden Gründen dagegen Widerspruch einlegen [...], daß sie betreffende Daten verarbeitet werden; dies gilt nicht bei einer im einzelstaatlichen Recht vorgesehenen entgegenstehenden Bestimmung. Im Fall eines berechtigten Widerspruchs kann sich die vom für die Verarbeitung Verantwortlichen vorgenommene Verarbeitung nicht mehr auf diese Daten beziehen;». Das DSG enthält ein analoges Recht in der Bestimmung von Art. 12 Abs. 2 lit. b DSG, der die Bearbeitung von Daten einer Person gegen deren ausdrücklichen Willen bei fehlender Rechtfertigung verbietet.

47 Die datenschutzrechtliche Ausgangslage ist somit in der Schweiz analog derjenigen in der EU. Der eidgenössischer Datenschutz- und Öffentlichkeitsbeauftragte vermutet denn auch, dass ein Schweizer Gericht in einer vergleichbaren Situation zum gleichen Schluss kommen würde ${ }^{81}$.

\section{Grundrechte}

48 Ebenfalls vergleichbar mit der Lage in der EU präsentiert sich die grundrechtliche Situation in der Schweiz. Die im Urteil genannten
79 nzz.ch vom 30. Mai 2014 (Zu früh gefreut); NZZ am Sonntag vom 1. Juni 2014, Nr. 22, S. 2 (Herkules-Aufgabe für Google).

80

Bundesgesetz über den Datenschutz vom 19. Juni
1992 (DSG, SR 235.1).

81 computerworld.ch vom 19. Mai 2014 (Thür: EUUrteil gegen Google auch für die Schweiz relevant). 
Grundrechte aus den Art. $7^{82}$ und $8^{83}$ der EUGrundrechtecharta entsprechen Art. 13 der Bundesverfassung ${ }^{84}$, wonach jede Person Anspruch auf Achtung ihres Privat- und Familienlebens, ihrer Wohnung, ihres Brief-, Postund Fernmeldeverkehrs sowie auf Schutz vor Missbrauch ihrer persönlichen Daten hat.

Der nur in den Schlussanträgen des Generalanwalts berücksichtigte und vom EuGH nicht beachtete Art. 11 der EU-Grundrechtecharta ${ }^{85}$ entspricht Art. 16 und 17 BV, welche die Meinungs-, Informations- und Medienfreiheit gewährleisten. Sowohl in der EU-Grundrechtecharta wie in der BV wird explizit auch die Empfangsfreiheit genannt. Art. 16 Abs. 3 BV hält fest, dass jede Person das Recht hat, «Informationen frei zu empfangen, aus allgemein zugänglichen Quellen zu beschaffen und zu verbreiten.» Nach Art. 11 Abs. 1 der EUGrundrechteverordnung schliesst das Recht auf freie Meinungsäusserung «die Freiheit ein, Informationen und Ideen ohne behördliche Eingriffe und ohne Rücksicht auf Staatsgrenzen zu empfangen und weiterzugeben.»

Der ebenfalls nur vom Generalanwalt berücksichtigte Art. 16 der EU-Grundrechtecharta ${ }^{86}$ anerkennt die unternehmerische Freiheit und

entspricht damit Art. $27 \mathrm{BV}$, der die Wirtschaftsfreiheit gewährleistet.

\section{Rechtfertigungsgründe}

51 Sowohl bei einer rein datenschutzrechtlichen Betrachtungsweise als auch unter Berücksichtigung der Grundrechte stellt sich die Frage nach einer Rechtfertigung der Datenbearbeitung bzw. des Eingriffs in die Privatsphäre. Im Datenschutzrecht sind die Rechtfertigungsgründe in Art. 13 DSG festgehalten und im Verfassungsrecht wird die Zulässigkeit von Grundrechtseinschränkungen nach Art. 36 BV beurteilt. Zentral ist in jedem Fall die Informationsfreiheit; sei dies in Form des überwiegenden öffentlichen Interesses als Rechtfertigungsgrund gemäss Art. 13 Abs. 1 DSG oder im Rahmen des Schutzes von Grundrechten Dritter nach Art. 36 Abs. 2 BV.

Letztlich ist somit die vom Generalanwalt vorgenommene Abwägung zwischen dem Interesse des Einzelnen an der Nichtverbreitung sensibler Informationen und dem Interesse der Allgemeinheit am Zugang zu Informationen auch für die Beurteilung des Falles nach schweizerischem Recht zentral. Für diese Beurteilung musste der Generalanwalt hypothetisch beurteilen, welche Folgen die Bejahung

84

Art. 7 EU-Grundrechtecharta (Achtung des Privatund Familienlebens): «Jede Person hat das Recht auf Achtung ihres Privat- und Familienlebens, ihrer Wohnung sowie ihrer Kommunikation.»

83 Art. 8 EU-Grundrechtecharta (Schutz personenbezogener Daten):

«(1) Jede Person hat das Recht auf Schutz der sie betreffenden personenbezogenen Daten.

(2) Diese Daten dürfen nur nach Treu und Glauben für festgelegte Zwecke und mit Einwilligung der betroffenen Person oder auf einer sonstigen gesetzlich geregelten legitimen Grundlage verarbeitet werden. Jede Person hat das Recht, Auskunft über die sie betreffenden erhobenen Daten zu erhalten und die Berichtigung der Daten zu erwirken.

(3) Die Einhaltung dieser Vorschriften wird von einer unabhängigen Stelle überwacht.»
Bundesverfassung der Schweizerischen Eidgenossenschaft vom 18. April 1999 (BV, SR 101).

85 Art. 11 EU-Grundrechtecharta (Freiheit der Meinungsäußerung und Informationsfreiheit):

«(1) Jede Person hat das Recht auf freie Meinungsäußerung. Dieses Recht schließt die Meinungsfreiheit und die Freiheit ein, Informationen und Ideen ohne behördliche Eingriffe und ohne Rücksicht auf Staatsgrenzen zu empfangen und weiterzugeben.

(2) Die Freiheit der Medien und ihre Pluralität werden geachtet.»

86 Art. 16 EU-Grundrechtecharta (Unternehmerische Freiheit): «Die unternehmerische Freiheit wird nach dem Unionsrecht und den einzelstaatlichen Rechtsvorschriften und Gepflogenheiten anerkannt.» 
eines Rechts auf Vergessenwerden nach sich ziehen würde. Er hat sich dieser schwierigen Aufgabe angenommen und dem Gerichtshof davon «ab[ge]raten, in seinem Urteil zu dem Ergebnis zu gelangen, dass diese einander widerstreitenden Interessen im jeweiligen Einzelfall auf zufriedenstellende Weise in ein Gleichgewicht gebracht werden können und dass die Entscheidung dem Internetsuchmaschinen-Diensteanbieter überlassen bleibt» ${ }^{87}$. Er hat darauf hingewiesen, dass derartige Verfahren zur Meldung und Entfernung «wahrscheinlich entweder zu einer automatischen Löschung von Links zu beanstandeten Inhalten oder zu einer von den beliebtesten und wichtigsten Internetsuchmaschinen-Diensteanbietern nicht zu bewältigenden Anzahl von entsprechenden Anträgen führen» würden ${ }^{88}$.

Während noch nicht klar ist, wie Google mit den Löschanträgen im Einzelfall umgehen wird, ist aus heutiger Sicht ein Teil der Befürchtungen des Generalanwalts bereits eingetreten. Auch wenn Google jedes Jahr hohe Gewinne erzielt und eine grosse Anzahl von Juristen $^{89}$ beschäftigen kann, dürfte bei einer Flut von bis zu $12^{\prime} 000$ Anträgen pro $\mathrm{Tag}^{90} \mathrm{die}$ Versuchung gross sein, nach dem Prinzip «im Zweifel löschen» zu verfahren ${ }^{91}$. Somit ist

Schlussanträge des Generalanwalts vom 25. Juni 2013, Rn. 133.

88 Schlussanträge des Generalanwalts vom 25. Juni 2013, Rn. 133.

89 Siehe dazu Carlo Piltz, delegedata.de vom 4. Juli 2014 (Recht auf Vergessen - Warum Google nicht überreagiert): «Natürlich wäre es wünschenswert, wenn die Abwägung von im Presse- oder Datenschutzrecht versierten Juristen vorgenommen wird. Eine Pflicht hierzu, besteht aber nicht».

90

nzz.ch vom 31. Mai 2014 (12’000 Anträge am ersten Tag) sowie nzz.ch vom 8. Juli 2014 (Die Schatten des Rechts auf Vergessen): «Rund $1000 \mathrm{Be}-$ gehren treffen täglich ein. Sie summierten sich im Juni auf 70'000, wobei insgesamt $270^{\prime} 000$ Websites betroffen sind». auch der Beurteilung des Generalanwalts zuzustimmen, wonach mit diesem Urteil «entscheidende Rechte wie die Freiheit der Meinungsäußerung und die Informationsfreiheit geopfert» wurden. Zumindest nach schweizerischem Recht müsste die Abwägung somit anders ausfallen, d.h. Suchmaschinenbetreiber können nicht dazu verpflichtet werden, rechtmässige Informationen aus den Ergebnislisten zu entfernen. Etwas anderes gilt für die Entfernung von eindeutig rechtswidrigen Inhalten $^{92}$.

\section{Kartellrecht}

54 Sollte Google tatsächlich dazu übergehen, bei der Bearbeitung von Löschanträgen Grosszügigkeit walten zu lassen und im Zweifel eher zu löschen, könnte dies wiederum eine kartellrechtliche Problematik zur Folge haben. Eine Webseite kann sensible Informationen über eine Person enthalten und gleichzeitig in den Augen einer anderen Person oder eines Unternehmens wichtig und richtig sein. Sollte die Seite auf Antrag der erstgenannten Person aus der Trefferliste verbannt werden, kann dies die andere Person in ihrem Wettbewerb beeinträchtigen. An diesem Beispiel zeigt sich, wie schwierig die vom EuGH auf Google übertragene Richterfunktion werden kann, zumal ein kartellrechtlicher Anspruch auf Vorkommen

91 Google hält in den häufig gestellten Fragen indessen fest, dass «wir jeden Antrag individuell prüfen und zwischen dem Recht des Einzelnen auf Schutz seiner personenbezogenen Daten und dem Recht der Öffentlichkeit auf Zugang zu diesen Informationen und ihrer Verbreitung abwägen müssen». Vgl. aber Carlo Piltz, delegedata.de vom 4. Juli 2014 (Recht auf Vergessen - Warum Google nicht überreagiert): «Nur «in besonders gelagerten Fällen〉 könne ein Ausgleich der verschiedenen Interessen etwa von der Art der Informationen oder deren Sensibilität für das Privatleben der betroffenen Person abhängen. Dies bedeutet freilich nichts anderes, als dass im Zweifel die Löschung der Links in der Ergebnisliste vorgenommen werden muss».

92 Suchmaschinenhaftung, Diss. Bern 2012, $\S 12, \mathrm{I}, 3$. 
in den Suchergebnissen einer marktbeherrschenden Suchmaschine besteht ${ }^{93}$.

\section{Ausblick}

\section{Weiterzug an den EGMR}

55 Weil die durch das Urteil des EuGH beeinträchtigte Informationsfreiheit auch von der EMRK geschützt wird, stellt sich die Frage, ob ein Weiterzug des Urteils an den EGMR möglich wäre. Nach Art. 6 des Vertrages von Lissabon $^{94}$ tritt die EU der EMRK bei. Der Beitritt ist allerdings bis zum heutigen Zeitpunkt noch nicht erfolgt ${ }^{95}$, sodass ein direkter Weiterzug nicht ohne weiteres möglich erscheint.

Das Urteil des EuGH erging im Rahmen eines Vorabentscheidungsverfahrens, d.h. es braucht nach diesem noch die Umsetzung durch das vorlegende Gericht. Hier soll nicht die Frage untersucht werden, ob eine grundrechtswidriges Urteil des EuGH eine Bindungswirkung entfalten kann. Möglich wäre aber ein Weiterzug der Entscheidung der Audiencia Nacional an den EGMR. Weil davon auszugehen ist, dass sich die Audiencia Nacional an die Vorgaben des EuGH halten wird, ist mit einem die Informationsfreiheit übermässig einschränkenden Entscheid zu rechnen, der durch den EGMR anders beurteilt werden könnte als durch den EuGH.

\section{Rückzug aus EU-Staaten}

57 Da die Google Inc. ihren Sitz in Kalifornien hat und das grösste Forschungszentrum für Europa, den mittleren Osten und Afrika in Zürich betreibt ${ }^{96}$, wäre es für das Unternehmen eine Überlegung wert, sich aus den EU-Staaten zurückzuziehen und alle Aktivitäten in Nicht-EU-Raum zu verlegen. Auch wenn eine solche Lösung für das stolze Nicht-EU-Land Schweiz auf den ersten Blick interessant erscheint, ist dieses Vorgehen nicht zu empfehlen: Die Revision der Datenschutz-Richtlinie sieht vor, dass das Datenschutzrecht schon dann anwendbar ist, wenn eine Dienstleistung in der Union angeboten wird ${ }^{97}$.

\section{Korrektur durch den Gesetzgeber}

58 Im Urteil des EuGH wird erwähnt, dass sich die EU-Kommission entgegen ihrer nach dem Urteil geäusserten Meinung ${ }^{98}$ gegen ein Recht
93 Suchmaschinenhaftung, Diss. Bern 2012, § 11, III.

94 Vertrag von Lissabon zur Änderung des Vertrags über die Europäische Union und des Vertrags zur Gründung der Europäischen Gemeinschaft, unterzeichnet in Lissabon am 13. Dezember 2007 (AB1. C 306 S. 1-229).

95 Vgl. dazu die Pressemitteilung des Europarats vom 5. April 2013 (Meilenstein bei Verhandlungen über Beitritt der EU zur Europäischen Menschenrechtskonvention erreicht).

96 Google Zurich, Accomplishments: «We’re Google's largest engineering office in Europe, the Middle East and Africa.»

97 Art. 3 Abs. 2 lit. a des Kommissionsvorschlags vom 25. Januar 2012 für eine Verordnung des Europäischen Parlaments und des Rates zum Schutz natürlicher Personen bei der Verarbeitung personenbezogener Daten und zum freien Datenverkehr (Datenschutz-Grundverordnung): «Die Verord- nung findet Anwendung auf die Verarbeitung personenbezogener Daten von in der Union ansässigen betroffenen Personen durch einen nicht in der Union niedergelassenen für die Verarbeitung Verantwortlichen, wenn die Datenverarbeitung a) dazu dient, diesen Personen in der Union Waren oder Dienstleistungen anzubieten,».

98 euronews.com vom 13. Mai 2014 (Gerichtsurteil: Auch Google muss vergessen können): «Kommissionssprecherin Mina Andreeva sagte, die Europäische Kommission begrüße die Entscheidung des Gerichts: «Auch eine Suchmaschine wie Google muss sich also europäischem Recht beugen. Dies ist ein gutes Urteil, nicht nur für die Kommission, sondern auch für die Bürger, deren Daten nun besser geschützt sind.»») Siehe auch euronews-Beitrag vom 13. Mai 2014 (EU court says Google must honour 'right to be forgotten', Aussage der Kommissionssprecherin bei 0:40). 
auf Vergessen ausgesprochen hat. Gemäss Urteilstext ist nach ihrer Auffassung die Frage, ob «die betroffene Person vom Suchmaschinenbetreiber verlangen kann, von der Ergebnisliste, die im Anschluss an eine anhand ihres Namens durchgeführte Suche angezeigt wird, Links zu von Dritten rechtmäßig veröffentlichten Internetseiten mit wahrheitsgemäßen Informationen über sie zu entfernen, weil diese Informationen ihr schaden können oder weil sie möchte, dass sie nach einer gewissen Zeit 〈vergessen〉 werden ${ }^{99}{ }^{9}$ u verneinen ${ }^{100}$.

Somit erscheint denkbar, dass das überschiessende Urteil des EuGH im Rahmen der laufenden Revision der EU-Datenschutzrichtlinie korrigiert wird ${ }^{101}$. Da Google die beanstandeten Suchtreffer nur auf den europäischen Länderseiten ausblendet und die Nutzer demzufolge wohl vermehrt nur noch die unzensierte Version via google.com abrufen werden, erscheint das Urteil auch unabhängig von der grundrechtlichen Problematik ungeeignet zum

99 Urteil des EuGH vom 13. Mai 2014, Rn. 89.

${ }^{100}$ Urteil des EuGH vom 13. Mai 2014, Rn. 90: «Nach Auffassung von Google Spain, Google Inc., der griechischen, der österreichischen und der polnischen Regierung sowie der Kommission ist diese Frage zu verneinen. Google Spain, Google Inc., die polnische Regierung und die Kommission machen insoweit geltend, Art. 12 Buchst. b und Art. 14 Abs. 1 Buchst. a der Richtlinie 95/46 gewährten den betroffenen Personen nur unter der Voraussetzung Rechte, dass die betreffende Verarbeitung nicht den Bestimmungen der Richtlinie entspreche, oder aus überwiegenden, schutzwürdigen, sich aus ihrer besonderen Situation ergebenden Gründen, und nicht bereits, weil die Verarbeitung ihnen ihrer Auffassung nach schaden könne oder sie möchten, dass die Daten, die Gegenstand der Verarbeitung seien, dem Vergessen anheimfallen. Nach Auffassung der griechischen und der österreichischen Regierung hat sich die betroffene Person an den Herausgeber der Website zu wenden.»

${ }^{101}$ In eine andere Richtung weist allerdings die Äusserung des deutschen Bundesinnenministers de Maizière, der in einer Pressemitteilung vom 30. Juni 2014 erklärte: «Der Europäische Gerichtshof
Schutz des vom EuGH in den Vordergrund gerückten Rechts auf Schutz personenbezogener Daten.

60 In diesem Zusammenhang sei auch auf die digitale Agenda der EU-Kommission hingewie$\operatorname{sen}^{102}$. Diese enthält Aussagen wie z.B.: «Die Verbraucher erwarten zu Recht, dass sie auf Online-Inhalte zumindest genauso leicht wie in der Offline-Welt zugreifen können» ${ }^{103}$ und beklagt mehrfach die führende Rolle der USA im Online-Bereich ${ }^{104}$. Vor diesem Hintergrund dürfte das jüngste Urteil des EuGH den Plänen der EU-Kommission einen Bärendienst erwiesen haben.

\section{Alternative Lösungsmöglichkeiten}

61 Auch wenn nach der vorliegend vertretenen Auffassung der beanstandete Suchtreffer nicht entfernt werden darf, ist das Anliegen der betroffenen Person nachvollziehbar. Auch hier gilt jedoch, dass man nicht den Boten töten ${ }^{105}$, sondern das Problem an der Wurzel anpacken

hat uns in seiner Entscheidung zu Internetveröffentlichungen und zum Recht auf Vergessen einen Auftrag erteilt, den wir umsetzen müssen.»

102 Mitteilung der Kommission vom 19. Mai 2010 an das Europäische Parlament, den Rat, den europäischen Wirtschafts- und Sozialausschluss und den Ausschuss der Regionen: Eine Digitale Agenda für Europa (KOM[2010]245 endgültig).

103 Eine Digitale Agenda für Europa, S. 8.

${ }^{104}$ Eine Digitale Agenda für Europa, S. 5 f.: «Aufgrund fehlender legaler Angebote und fragmentierter Märkte gibt es in den USA heute viermal so viele Musik-Downloads wie in der EU»; S. 11: «die Europäer finden es zudem oft einfacher, eine grenzüberschreitende Online-Transaktion mit einem US-amerikanischen Anbieter auszuführen als mit einem Verkäufer aus einem anderen europäischen Land»; S. 26: «Im Vergleich zu wichtigen Handelspartnern wie den USA ist in Europa der IKT-Anteil an den FuE-Ausgaben nicht nur viel geringer (17\% gegenüber $29 \%$ ), sondern beträgt auch in absoluten Zahlen nur etwa $40 \%$ des USBudgets».

105 Shakespeare, The Tragedie of Anthonie, and Cleopatra: «Messenger: Gratious Madam, I that do bring the newes, made not the match». 
sollte - jedenfalls dann, wenn die Rechtmässigkeit eines Suchergebnisses umstritten ist.

Will man von Suchmaschinen verlangen, bestimmte Inhalte generell oder nach einer gewissen Zeit zu «vergessen», sollte man die betreffenden Websites entsprechend konfigurieren. So steht es z.B. jedem Betreiber eines Online-Archivs offen, den gesamten Inhalt ${ }^{106} \mathrm{o}$ der Datenbestände mit einem gewissen Alter ${ }^{107}$ oder auch nur die Namen von Personen ${ }^{108}$ nicht mehr indexieren zu lassen. Sollte der Gesetzgeber in der EU oder in der Schweiz dies als richtig erachten, müsste er solche Überlegungen auch in die laufenden Revisionsarbeiten miteinbeziehen. Darüber hinaus besteht auch ein Interesse der Webseitenbetreiber, insbesondere von Medien ${ }^{109}$, selber darüber zu entscheiden, unter welchen Umständen ihre Seiten in den Ergebnislisten von Suchmaschinen erscheinen.

Im Fall, den der EuGH zu entscheiden hatte, kommt hinzu, dass die fragliche Information zunächst nur in der gedruckten Version der Zeitung existierte und erst später durch den Herausgeber auch online verfügbar gemacht wurde $^{110}$. Der Gerichtshof hätte m.E. auch die
Frage aufgreifen sollen, ob der Verstoss gegen das Datenschutzrecht nicht in der nachträglichen Digitalisierung und Verfügbarmachung durch den Herausgeber zu sehen ist. Die Vorinstanz hatte die Beschwerde, soweit sie sich gegen den Herausgeber richtete, mit der Begründung zurückgewiesen, «die Veröffentlichung der betreffenden Informationen durch diese Gesellschaft sei rechtlich gerechtfertigt gewesen, da sie auf Anordnung des Arbeitsund Sozialministeriums und mit dem Ziel einer höchstmöglichen Publizität der Zwangsversteigerung und somit einer höchstmöglichen Zahl an Bietern erfolgt sei.» ${ }^{111} \mathrm{Sie}$ hat sich aber offenbar nicht dazu geäussert, ob und ggf. mit welcher Begründung die spätere Digitalisierung und Onlineveröffentlichung zu rechtfertigen ist.

\section{Fazit}

64 Das Google-Urteil des EuGH verstösst gegen die Informationsfreiheit, die sowohl von der EU-Grundrechtecharta als auch durch die EMRK und BV garantiert wird. Dieser Verstoss lässt sich nicht mit dem überwiegenden Interesse einer Privatperson rechtfertigen, solange es sich - wie im vorliegenden Fall -
${ }^{106}$ Die Indexierung aller Inhalte durch sämtliche Suchmaschinen wird unterbunden, wenn die Datei robots.txt im Stammverzeichnis der Domain folgende Anweisung enthält: «User-agent: * Disallow: /».

${ }^{107}$ Die einfachste Möglichkeit zur Nichtindexierung von älteren Inhalten besteht darin, diese in anderen Verzeichnissen abzulegen und die Indexierung dieser Verzeichnisse zu unterbinden, z.B. mit der folgenden Anweisung: «User-agent: * Disallow: /old/».

${ }^{108}$ Die Unterbindung der Indexierung von Namen erfolgt nicht über die Datei robots.txt, sondern über entsprechende Anweisungen im HTML-Code. Diese Möglichkeit wird, soweit ersichtlich, von Google noch nicht unterstützt, aber von Yahoo (Introducing Robots-Nocontent for Page Sections) und der russischen Suchmaschine Yandex (Using HTML tags, <noindex> tag). Ob im Nachgang zum Urteil des EuGH auch Google diese Möglichkeit einführen wird, ist nicht bekannt. Im Janr 2009 hat sich der Leiter des Webspam-Teams von Google dagegen ausgesprochen. Siehe dazu den Tweet von Matt Cutts vom 6. Juli 2009: «we looked at how many sites use robots nocontent on the web and it was miniscule, so we decided not to do it».

${ }^{109}$ Siehe dazu theguardian.com vom 2. Juli 2014 (EU's right to be forgotten: Guardian articles have been hidden by Google): «Publishers must fight back against this indirect challenge to press freedom, which allows articles to be 'disappeared'. Editorial decisions belong with them, not Google».

${ }^{110}$ Im Urteil des EuGH wird dies nicht erwähnt, aber in den Schlussanträgen des Generalanwalts vom 25. Juni 2013, Rn. 18.

${ }^{111}$ Urteil des EuGH vom 13. Mai 2014, Rn. 16. 
um eine rechtmässig veröffentlichte Information handelt. Mit dem Urteil wird Suchmaschinenbetreibern eine Richterfunktion zugewiesen, die in einem Rechtsstaat nicht an Private delegiert werden darf ${ }^{112}$.

Weil der EuGH verschiedene Grundrechte auszublenden scheint, lohnt sich ein Blick auf die Schlussanträge des Generalanwalts, die einen sinnvollen Lösungsvorschlag sowohl für das schweizerische wie auch das europäische Recht enthalten. Insofern kann der Einschätzung des EDÖB, wonach ein Schweizer Gericht in einer vergleichbaren Situation zum gleichen Schluss kommen sollte, nicht gefolgt werden. Eine Pflicht zur Entfernung von Suchergebnissen trifft die Suchmaschinenbetreiber m.E. nur dann, wenn es sich um offensichtlich rechtswidrige Inhalte handelt ${ }^{113}$.

112 Siehe auch Carlo Piltz, delegedata.de vom 4. Juli 2014 (Recht auf Vergessen - Warum Google nicht überreagiert): «Wollte man verlangen, dass Google sich bei der Prüfung auch in die Lage der Webseitenbetreiber und der Öffentlichkeit versetzt, so würde man dem Suchmaschinenbetreiber eine unabhängige Rolle zusprechen wollen, die er aber nicht besitzt.»

113 Suchmaschinenhaftung, Diss. Bern 2012, $\S 12, \mathrm{I}, 3$. 